\title{
Advances in Mapping Periodic Structural Modulations of Atomic Lattices
}

Ismail El Baggari ${ }^{1}$, Robert Hovden ${ }^{2}$, David J. Baek ${ }^{3}$, Alemayehu S. Admasu ${ }^{4,5}$, Jaewook Kim ${ }^{4,5}$, SangWook Cheong ${ }^{4,5}$ and Lena F. Kourkoutis ${ }^{2,6}$

${ }^{1 .}$ Department of Physics, Cornell University, Ithaca NY 14853, USA.

2. School of Applied and Engineering Physics, Cornell University, Ithaca, NY 14853, USA.

3. School of Electrical and Computer Engineering, Cornell University, Ithaca NY 14853,USA.

4. Department of Physics and Astronomy, Rutgers University, Piscataway NJ 08854, USA.

5. Rutgers Center for Emergent Materials, Rutgers University, Piscataway NJ 08854, USA.

${ }^{6 .}$ Kavli Institute at Cornell, Cornell University, Ithaca NY 14853, USA.

Charge density waves (CDW) are periodic modulations of the electron density and the lattice and often compete with high- $T_{c}$ superconductivity or ferromagnetism in oxides [1]. To date, linking the local structure of $\mathrm{CDW}$ to macroscopic phenomena remains a formidable challenge. Fortunately, subangstrom resolution in annular dark field scanning transmission electron microscopy (ADF STEM) and significant progress in quantitative analysis of ADF images promise direct understanding of structural modulations [2]. In this work, we develop a method to detect and classify atomic columns based on local information such as position, intensity and a priori structural and chemical constraints. We show that supplying such constraints is crucial for successful classification of columns for large field of views and for ADF images with inhomogeneous intensities. Here, we investigate room temperature $\left(\mathrm{T}_{\mathrm{c}}>500 \mathrm{~K}\right)$ charge modulated $\mathrm{Bi}_{0.5}(\mathrm{Sr}, \mathrm{Ca})_{0.5} \mathrm{MnO}_{3}$ [3]. A [010] oriented TEM sample was prepared from a single crystal by focused ion beam lift-out. STEM imaging was performed at $300 \mathrm{kV}$ on a FEI Titan Themis. Crucially, scan distortions are minimized by cross-correlating, aligning and averaging 30 rapidacquisition images ( $1 \mu$ s per pixel in 1024 pixel images).

The Fourier transform of the real space ADF image (Fig. 1) displays Bragg peaks decorated by satellite peaks that correspond to periodic modulations of the atomic lattice. The presence of these peaks in two orthogonal directions reveals that the modulation forms $90^{\circ}$ twin domains. In order to gain a real-space understanding of the modulation, the corresponding ADF image is shown in Figure 1b. Fourier filters of increasing size around the 202 Bragg spot (Fig 1c) reveal emerging modulations of the lattice and a complex domain structure.

Large field of view ADF images are necessary to resolve satellite peaks and reveal the CDW domain structure and coherence length. This, however, introduces challenges in direct quantification of atomic distances and bond modulations. Particularly, inhomogeneous contrast and overlapping intensities (Fig. 1b, 2a) limit the possibility to delineate the $\mathrm{Bi} / \mathrm{Sr} / \mathrm{Ca}(\mathrm{A})$ and $\mathrm{Mn}(\mathrm{B})$ columns over large fields of view using the intensity metric alone. Here, we develop a robust method that combines intensity mapping and prior knowledge about the coordination environment to detect and classify atomic columns correctly. First, atomic peaks are detected by cross-correlating a Gaussian shape with the whole image and refining positions and intensities [2]. Then, $\mathrm{n}^{\text {th }}$ nearest-neighbors (NN) are computed and stored (Fig. 2b). The $\mathrm{NN}$ are the foundation for defining a chemical environment. In our case, every A atom should be coordinated by $4 \mathrm{~B}$ atoms (and vice versa) as seen in Figure $2 \mathrm{~d}$ inset. Using this method, we are able to separate the ADF image into a B and A sublattice (Fig. 2c,d). The FFTs of the B and A atomic positions recover the satellite peaks (white circle) and support a structural modulation as the periodic modulation. In conclusion, structural and chemical constraints pave the way for robust analysis of inhomogeneous ADF images and potentially high-throughput processing and machine learning of large datasets [4]. 
[1] M. Uehara et al., Nature 399, 560-563 (1999).

[2] X. Sang et al., Microscopy and Microanalysis 20, 06 (2014).

[3] J. C. Loudon et al., Phys. Rev. Lett. 94, 097202 (2005).

[4] We acknowledge support by the Packard Foundation. This work made use of the Cornell Center for Materials Research Shared Facilities supported through the NSF MRSEC program (DMR-1120296) and a NSF MRI award (DMR-1429155).
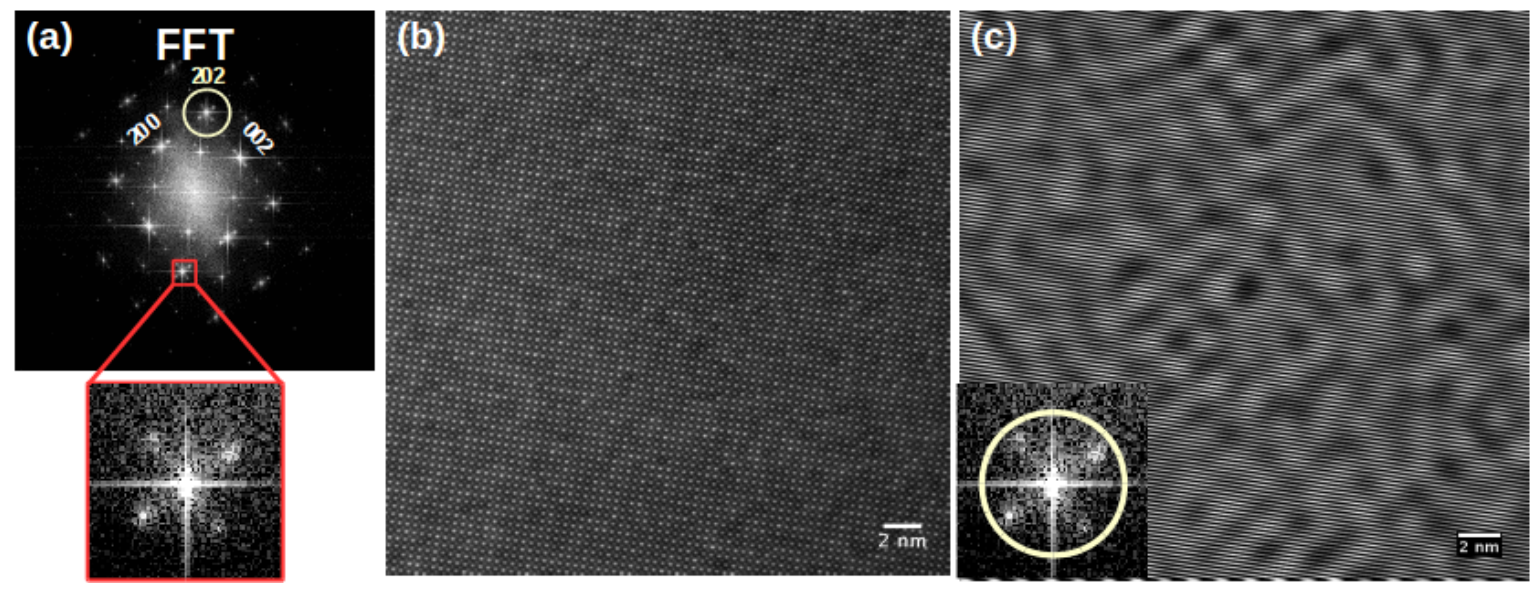

Figure 1. a, FFT of ADF image in (b) showing satellite peaks and twinning at the nanoscale. Bragg peaks are clearly decorated by satellite peaks (red box) corresponding to periodic modulations. b, Large field of view atomic-resolution ADF image exhibiting non uniform contrast and intensities. c, Fourier filtered image around the 202 spot exhibits complex modulations and domain patterns. The size of the mask was increased systematically (not shown) to unravel the contributions of the satellite peaks.
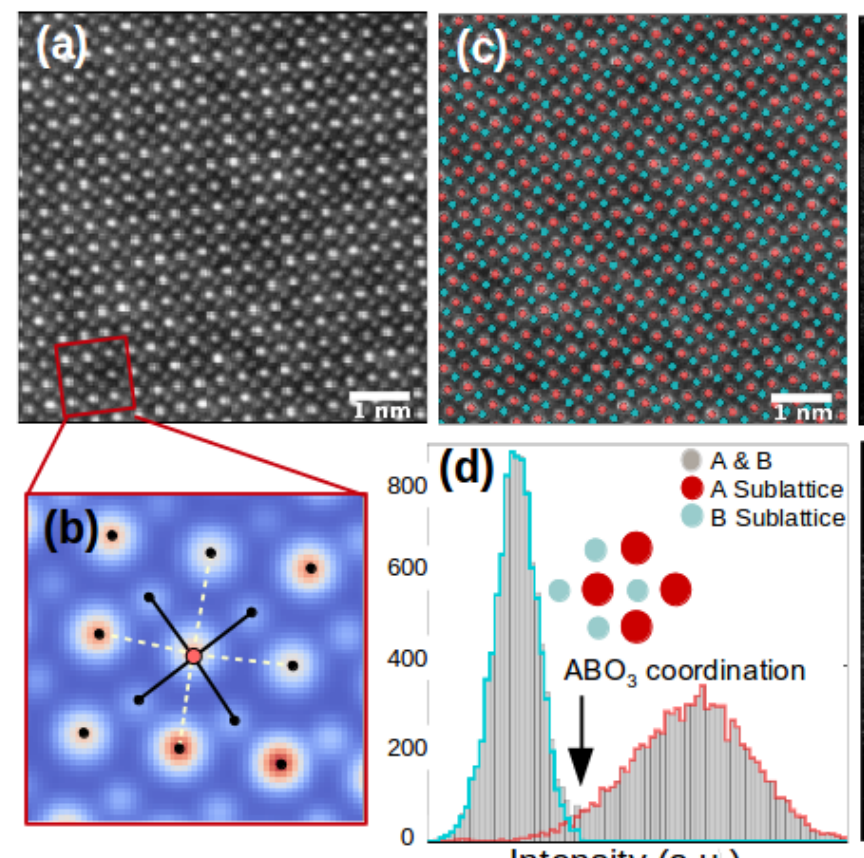

Intensity (a.u.)
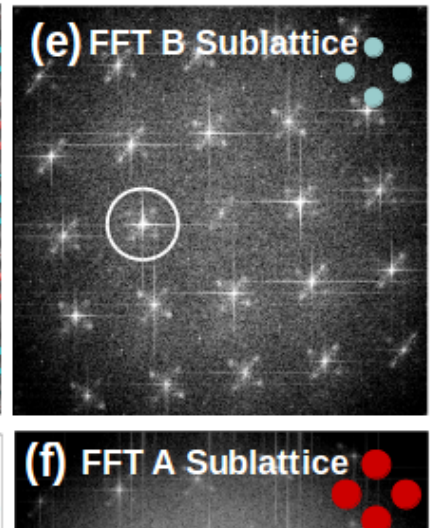

Figure 2. a, Representative section of an ADF image exhibiting inhomogeneous intensities. b, Gaussian fits to atomic columns. All atomic peak positions and intensities are detected (black dots). NN (black line) and Next NN (white dashed line) are computed for a given column (pink dot) in order to determine local coordination. d, Histogram of column intensities in gray shows overlap (black arrow) for different sublattices. Using the structural constraint (inset), the A (red) and B (turquoise) sublattices are delineated as shown in c. The red (turquoise) envelope is intensity histogram of A (B). e-f), Robust separation of sublattices reveals contribution of each sublattice (species) to CDW modulations. Both A and B contribute to the structural modulation as seen in satellite peaks circled in white. 